\title{
The effect of surface treatments on the Nanomechanical properties of human hair
}

\author{
Nan Wang ${ }^{1}$, Richard Barfoot ${ }^{2}$, Michael Butler $^{3} \&$ Colm Durkan ${ }^{1 *}$ \\ ${ }^{1}$ : Nanoscience, University of Cambridge, $11 \mathrm{JJ}$ Thomson Avenue, Cambridge, CB3 0FF, UK \\ 2: Unilever R\&D, Port Sunlight, Quarry Road East, Bebington, Wirral, CH63 3JW, UK \\ 3: Unilever R\&D, Colworth Science Park, Sharnbrook, Bedford, MK44 1LQ, UK \\ *: corresponding author, email: $\underline{\mathrm{cd} 229 @ \text { eng.cam.ac.uk }}$
}

KEYWORDS: hair; AFM; surface; contact mechanics; Surface potential.

\begin{abstract}
The structural properties of hair are largely determined by the state of the surface. Advanced imaging modes of Atomic force microscopy (AFM), where the surface mechanics can be correlated with surface topography have been used to spatially map variations in hair surfaces following chemical and mechanical treatments. Through analysis of multilayered data obtained in this way, we show that the processes of bleaching and combing of hair not only alter the surface roughness, but also alter the mechanical stiffness, adhesion properties and surface potential of hair, in terms of the mean values and their distributions. These treatments are shown to have a significant effect on the nanoscale surface properties consistent with what has previously been observed at the macroscopic fiber-level scale.
\end{abstract}

\section{Introduction}

Optimization of hair quality is a fundamental problem faced by billions of people on a daily basis. This is a particularly intricate issue as hair is arguably the most complex composite biological material in existence[1]. As a result of its multi-component and hierarchical structure, the properties (chemical and physical) of individual hair fibers depend on environmental factors such as humidity, temperature, air quality and exposure to sunlight [1], [2], as well as factors unique to the individual 
such as diet and haircare regimen. These factors, coupled with intrinsic physiological variations due to race have led to the continual development of a plethora of haircare formulations aimed at tackling these issues. With recent legislation [3] requiring substantiation of claims for cosmetic/personal care products, it is becoming ever more important to have objective measures in place to determine hair quality and the actual vs intended effect of specific formulations. This is hampered from the point of view that there is no definitive measure of hair quality, nor any consensus as to what it is apart from quite general individual and entirely subjective preferences regarding shine, strength, color, volume, and overall manageability. Our studies show that even simple treatments such as bleaching and combing can lead to significant modification of and damage to the hair surface, which will then have a detrimental effect on the overall fiber properties. It must be pointed out though that macroscopic measurements reveal information about the fibre as a whole, whereas our measurements are surface-specific.

Strands of hair are each covered in typically 5-10 layers of interleaved cells, called cuticles, which have a high keratin content. Cuticles are typically around $300 \mathrm{~nm}-600 \mathrm{~nm}$ thick, 40-60 microns long and form an overlapping structure with a period between 5-10 microns. They are covered by a hydrophobic fatty acid layer, known as the B-layer, typically 5-7 nm thick, comprising 18-Methyl eicosanoic acid. This acts as a protective barrier against water penetration[1]. This layer is covalently bound to the layer underneath, which is a lipo-protein complex called the epicuticle, which is $10-15 \mathrm{~nm}$ thick. In turn, this layer is supported by the A-layer, the exocuticle and the endocuticle, respectively, as illustrated in Figure 1. In between the cuticles is the cell membrane complex that acts as a glue holding the cuticles together. The top few layers can be easily damaged either mechanically or chemically, rendering the hair prone to chemical ingress which can ultimately compromise the structure of the entire strand. The condition of the cuticles on each strand is a determining factor of not only the aesthetic appearance, but also the mechanical properties and response of the hair to any given treatment.

In personal hair care, brushing/combing and coloring/bleaching are commonplace. These processes have the potential to significantly affect the hair structure. Of particular interest is how these treatments change hair properties, especially those of the surface. In recent years, there has been a significant amount of research to systematically determine the effect and efficacy of hair products in order to help develop improved formulations[5]. Studies have been focused on not only studying the microstructure, but also the nanostructure of hair strands, using a variety of characterization tools including scanning electron microscopy (SEM)[6], transmission electron microscopy (TEM), and atomic force microscopy (AFM) [7]. Efforts have also been made to study the effects of environmental or chemical damage and treatment, such as dyeing, bleaching, and polymer application [8-10]. Traditional measurements have tended to focus on the macroscopic mechanical properties of single hair fibers or on fiber bundles. It is well known that hair that has been treated either chemically or mechanically is softer and has a lower yield stress. What is less well known is whether there is any correlation between these macroscopic properties and the corresponding nanoscopic properties of the hair surface. Given that the diameter of hair is typically in the range 50-80 microns, it is not obvious that the surface mechanical properties would vary in the same way as the bulk fiber properties after treatment. 
Understanding the local nanomechanical properties of hair is a subject of growing interest as to a large extent they determine the macroscopically-observed properties. The vast majority of the mechanical property studies of human hair are on the macroscale and employ conventional methods such as tension, torsion and bending tests [11], [12]. In relatively recent times, a number of studies have been conducted on the elasticity of hair at the nanoscale using AFM [13-15].

Hybrid mode is an AFM technique where mechanical properties of a surface can be obtained together with topography data, which is what we concentrate on in this article. In hybrid mode, the tip-sample distance is modulated periodically in the range from a few $\mathrm{nm}$ to several $10 \mathrm{~s}$ of $\mathrm{nm}$, during which time the force on the tip is continually monitored at a high frequency, in our case $2 \mathrm{MHz}$. Previous attempts at carrying out such work were demonstrated over 15 years ago [16] but were only semi-quantitative due to nonlinearities in the sort of open-loop AFM scanners available at the time and through an inadequate knowledge of the tip's mechanical properties. Through the use of (i) closed-loop scanners, (ii) accurately-calibrated AFM cantilevers and (iii) an appropriate contact mechanics model, it is possible to obtain quantitative maps of topographical and mechanical properties of a sample surface with nm spatial resolution. This technique is similar in principle to peak-force tapping mode [17]. The concept behind the nanomechanical measurements is as shown in Figure 2. The cantilever is sinusoidally driven normal to the sample at a frequency in the range 0.8-1.2 kHz, far away from any cantilever or microscope resonances. The resulting deflection of the cantilever is monitored and compared with the expected deflection, and this information is analyzed within the context of a contact-mechanics model, resulting in quantitative measurements of the surface mechanical properties. In order to obtain accurate quantitative information, the cantilever stiffness needs to be measured, as does the tip geometry. The latter is challenging to obtain reliably, so we use it as our fitting parameter against a surface with known elastic modulus, namely Silicon (111).

Specifically, using hybrid mode, we can simultaneously measure the topography along with a number of mechanical properties. The quantities that we have found to be the most reliable at revealing differences between the different hair treatments consistently are the adhesion force between the tip and the hair, the elastic modulus and surface stiffness. The elastic modulus can only be determined if an appropriate model of the tip-sample contact mechanics is employed. There are multiple regimes found in contact mechanics as described by the Hertz [18], DMT [19] and JKR [20] models. As was shown by Maugis [21], these are all limiting cases of a more generalized model for contact mechanics that takes into account tip-sample adhesion forces and assumes elastic deformation of both tip and sample. The mathematics behind this model are rather unwieldy, so whenever appropriate, one of the above three models will be applied. In order to know which model is appropriate for any given situation, we estimate the value of the so-called Maugis parameter, $\lambda$, as given in equation 1 .

$$
\lambda=\frac{2.06}{D_{0}} \sqrt[3]{\frac{R W_{a d h^{2}}}{\pi E_{\text {tot }}^{2}}}
$$


where $R, E_{\text {tot }}, W_{\text {adh }}$ and $D_{0}$ are the tip radius of curvature, the combined Young's modulus of the tip-sample system, the tip-sample adhesion energy per unit area and the average atomic distance describing the system, which is empirically found to be $0.165 \mathrm{~nm}$ [21].

In the limit that $\lambda$ approaches 0 , corresponding to hard samples with low adhesion forces, the DMT model is an appropriate choice. Conversely, when $\lambda$ approaches infinity which corresponds to soft samples with high adhesion forces, the JKR model is used. The Hertz model can be applied in the case of zero tip-sample adhesion force, a regime which we do not encounter in studies of hair. We will use the numerical value of this parameter later to determine which contact mechanics model is the appropriate one for the systems we are studying.

In this paper, we present an investigation of local nanomechanical properties of the surface of human hair by hybrid mode atomic force microscopy. To explore differences in the effects of common processes, hair fibers that have been either chemically (by bleaching) or mechanically damaged (by combing) are studied and compared to untreated hair. The local stiffness, adhesion force and elastic modulus of the samples were investigated and the differences systematically studied.

\section{Experimental}

The strands of hair used in this study were dark brown European human hair obtained from International Hair Importers (New York, USA) and were provided by Unilever. Three types of hair samples were prepared: (a) untreated (b) bleached and (c) highly combed. The bleached sample has been prepared using a smooth mixture of $9 \%$ peroxide crème and powder bleach from L'Oréal (Paris, France) in a 2:1 w/w ratio, applied to dry hair and developed for $30 \mathrm{mins}$, then rinsed copiously, dried at room temperature, and all steps were repeated once. The highly combed hair has been combed 40000 times using an automated combing machine. Prior to measurement all sample types were washed using a 14\% Sodium Lauryl Ether Sulfate solution, rinsed copiously and then dried at room temperature. It is known that the topography and surface properties of hair vary significantly from one individual to another, as well as from one location to another as one moves along the hair shaft [22]. We have seen that the topography does indeed vary, but the mechanical properties tend to be more uniform and show much less variation along the shaft. In order to be able to draw more specific conclusions from this work, the bulk of the findings presented here are for hair from a single individual in order to rule out other factors influencing the surface properties. In order to demonstrate that our results are applicable more generally, we also show some data from other individuals that reveal similar trends.

AFM topography images were obtained using a Park XE-100 AFM and the hybrid images/data were collected using an NT-MDT Pro-M AFM with P5 and Hybrid controllers.

The basic imaging and nanomechanical probing experiments were carried out using tapping-mode cantilevers with stiffness in the range $20-40 \mathrm{~N} / \mathrm{m}$ and resonance frequency in the 
range $240 \mathrm{kHz}-440 \mathrm{kHz}$ (NSG30 probes from NTMDT). The Kelvin-Probe contact potential difference measurements were carried out using Electrimulti75-G platinum-coated tips, also from NTMDT. These have a stiffness and resonance frequency in the range 1-6 N/m and 60-90 kHz, respectively.

The methodology involves mounting several hair samples on a single sample plate using adhesive carbon tabs. Having all samples on the same mount makes it possible to directly compare the results obtained between the different hairs quickly, using the same AFM tip, and to quickly transfer from one microscope to another for complementary measurements.

\section{Results and Discussion}

\subsection{Hair topography and height profile.}

In Figure 3, we show the three different hair types as seen using optical microscopy, scanning electron microscopy (SEM) and AFM.

From Figure 3, the bleached hair, as well as the obvious color difference, has the appearance of having been damaged - the cuticle step edges are much less sharp than the untreated hair and there are occasional holes on the surface. The highly combed hair is severely damaged, with multiple holes typically 100-300 nm deep, roughened (laterally) cuticle edges and a significant amount of exposure of the endocuticle.

Figures $3 \mathrm{~h} \& 3 \mathrm{i}$ show AFM topographical images of chemically and mechanically damaged hair, whereas $3 \mathrm{~g}$ presents the data for untreated hair for comparison. It is well known that chemical and mechanical treatments cause observable cuticle damage. Hence in the case of damaged hair, along with failure and delamination in the endocuticular region, there is also evidence of fracture in the outer cuticle layers which then exposes the inner cuticle(s). The surface of bleached hair becomes highly porous as reported elsewhere [23].

To further highlight these differences, we have measured typical cross-sections of the surface topography of the different hair samples, and an indicative data set is shown in Figure 4. A number of numerical descriptors of the hair surface structure can be measured from these data, after [24], as indicated in Figure 5 and Table 1.

Table 1. Cuticular descriptors calculated for the example images shown in Figures 3 g-i.

\begin{tabular}{|l|l|l|l|}
\hline & Untreated & Bleached & Highly combed \\
\hline
\end{tabular}




\begin{tabular}{|l|l|l|l|}
\hline Step Height $(\mathrm{nm})$ & 500 & 360 & 255 \\
\hline Total length(um) & 9.0 & 9.2 & 7.2 \\
\hline $\begin{array}{l}\text { Cuticle linear density } \\
\left(\mathrm{mm}^{-1}\right)\end{array}$ & 111 & 109 & 139 \\
\hline
\end{tabular}

From Table 1, the untreated hair has the greatest cuticle step height; The highly combed hair has an average step height almost half that of the untreated hair; The bleached hair has less sharp cuticle edges and the steps are also lower than the untreated hair. For the combed hair, the cuticles will not have become thinner - material is removed in patches rather than consistently everywhere. Instead, they become compressed with the effect that some of the endocuticle (which has a gel-like consistency rather than the more solid exocuticle) has been squeezed out onto the neighboring surface.

Further small-scale images of the different hair types are shown in Figure 6, revealing that there are significant differences in appearance even at the sub-micron scale. AFM images of untreated hair reveal that the intrinsic morphology of the top layer (the B-layer) consists of round features roughly 50-100 nm across and with rms surface roughness of around 1-2 nm. We also occasionally observe striations along the hair axis (as shown in Figure $6(\mathrm{c})$ ), as reported by others and ourselves previously [6,7]. As pointed out in these references, the origin of these marks is not entirely clear but is likely due to either continuous cyclic expansion and shrinkage of the hair shaft due to variations in moisture levels and temperature resulting in stress fractures.

Bleaching is a process which lightens the shade of the hair by decolorizing the melanin in the hair shaft via an oxidation process. Melanin, which is brown, is transformed into oxymelanin which is colorless. The active agent in bleach is $\mathrm{H}_{2} \mathrm{O}_{2}$, and the oxidation reaction is catalyzed by the addition of ammonium carbonate, which is an alkali that has the dual function of neutralizing and then stripping the fatty acids present on the hair surface, allowing the hair cuticle to swell and hence the bleaching chemicals to penetrate into the hair shaft. It has been shown that the fatty acids are removed in patches a few tens of $\mathrm{nm}$ across, resulting in non-uniform penetration of bleach into the cuticle, and a porous appearance of the hair afterwards, consistent with our observations [25].

The process of combing hair results in physical stress on the hair fibers, which can cause the cuticles to flake and strip away. The combing process has clearly damaged the hair surface considerably, with a large number of holes in the cuticle upper layers, exposing the endocuticle, as discussed above.

\subsection{Hybrid mode AFM on hair samples}

Hybrid mode AFM has been used to further reveal the differences in the different hair samples caused by the various treatment regimes, beyond what topographic imaging can show. As 
explained in the introduction, in this mode of AFM, we periodically modulate the AFM tip up and down towards the sample, to the point where it lightly and reversibly indents the surface, staying within the elastic deformation regime. The deflection of the cantilever is measured using a linearized capacitance sensor, and the cantilever stiffness is determined through a measurement of the thermal noise spectrum. We find that in many cases, the topography images obtained using Hybrid mode have higher spatial resolution than conventional AFM images, especially when imaging soft samples such as hair. In Figure 7, we show topography, stiffness and adhesion force for the three different hair types, all for $1 \mu \mathrm{m}$ areas. There are clear differences in these properties between the samples, but on their own, the images do not appear to reveal much. Instead, we convert the images into histograms showing the distribution of the quantities of interest, which can then be compared across samples. One thing of note is that in the sample which has been bleached, we can clearly see some areas where the surface layer has been removed. Unaffected regions are labelled " $A$ " and regions where the 18-MEA layer has been removed are labelled " $B$ ". This is highlighted in Figure 7 (j) where we show the height cross-section along the line indicated in Figure $7(\mathrm{~d})$. Here, we can see that the thickness of the very top layer is of order $6-7 \mathrm{~nm}$. A surface topography analysis shows that the rms surface roughness is $1.48 \mathrm{~nm}$ in region $A$ and 1.74 $\mathrm{nm}$ in region $B$. In both cases, this surface roughness is as measured in a $200 \mathrm{~nm} \times 200 \mathrm{~nm}$ area, as over larger areas the long-range undulations of the surface start to dominate. The top of region $A$ consists of the tail end of the fatty acid, where the acid head is attached covalently to the crosslinked protein layer underneath. This protein layer is what we observe as region $B$. This structure is illustrated in Figure 7(k). We can also see from Figures 7 (d)-(f) that in the areas where the fatty acid has been removed (a region is indicated by a circle in these images), the stiffness is lower and the adhesion force between the tip and the sample is higher.

The distribution of surface stiffness, adhesion force and elastic modulus for the three hair types is shown in Figure 8. It is clear that the untreated hair has the largest variation of all three quantities. Both the highly-combed and bleached hair have a significantly reduced value of and variation in all properties. Hybrid mode reveals that the stiffness (the ratio of the applied force to the amount of deformation of the surface) is in the range $5-11 \mathrm{~N} / \mathrm{m}$, and via the DMT model this is found to correspond to an elastic modulus of the surface of the order 1-2 GPa, consistent with the expected value for keratin $[1,2]$. The adhesion force between the tip and the various hair surfaces is in the range $10-15 \mathrm{nN}$, most of which will be due to the capillary formation of a liquid bridge between them as a result of adsorbed water layers. In order to obtain a good fit between the measured tipsample force approach-retract characteristics, the fitting parameter we require is the tip radius of curvature, which we find to be typically in the range 7-15 $\mathrm{nm}$. The value of Maugis parameter we obtain for the measured Young's modulus of the sample and adhesion force is typically in the range 2-6, which is low enough that the DMT model is a reasonable fit. The AFM directly measures the sample deformation, $\delta$, (the difference between the distance the AFM cantilever is moved and the distance the tip moves when in contact with the sample), the tip-sample force, $F$ and the adhesion force, $F_{a d h}$. Using this information, the work of adhesion, $W_{a d h}$ and the Elastic modulus, $E_{\text {tot }}$ of the tip-sample system are calculated using the formulae in Equation 2. The AFM measures the cantilever deflection, $\Delta z$, from which we determine the force on the tip as $F=k \Delta z$. 


$$
W_{a d h}=\frac{F_{a d h}}{2 \pi R} \text { and } E_{t o t}=\frac{F+F_{a d h}}{\sqrt{R \delta^{3}}}, \text { where } \frac{1}{E_{t o t}}=\frac{3}{4}\left(\frac{1-v_{t i p}^{2}}{E_{t i p}}+\frac{1-v_{s}^{2}}{E_{s}}\right)
$$

Equation 2.

where $E_{t i p}, E_{s}, v_{t i p}$ and $v_{s}$ are the Young's modulus of the tip and sample and Poisson's ratio of the tip and sample, respectively. From this information, assuming Poisson's ratio for both tip and sample $=0.4$, we can calculate $E_{s}$. In Hybrid mode, we calculate or measure the values of $F, F_{a d h}$, $W_{a d h}, \delta, E_{s}$ and sample stiffness $(\delta / F)$ in real time and map the variation of these quantities simultaneously as we measure the surface topography. It must be pointed out that the data in Figure 8 is for single areas that were imaged. Measurements over a large number of areas reveal variations of up to $20 \%$ in the distribution and the mean of all quantities, but the above trends are consistently observed. Histograms for hair obtained from two additional source subjects are presented in Figure 9 showing identical trends.

Untreated hair has the highest elastic modulus and surface stiffness, i.e. all treatments we have carried out result in a softening of the surface. The bleaching process makes the hair stiffness more uniformly low. As a result of treatment, the changes we observe in mechanical and morphological properties of the surface are ultimately due to changes in the chemical species on the surface, either through removal of the fatty acid layer in the bleaching process, or coverage of the exocuticle by the endocuticle that is essentially dragged out from between cuticles during the combing process. These changes in chemical species will be evident using alternative measurement modalities, and as an example of this, we also used AFM to map the surface potential in Kelvin Probe Force Microscopy (KFPM) of the hair surfaces. It was shown some time ago that treatments alter the surface potential of hair [26- 27]. The surface of hair is intrinsically negatively charged and the process of combing/brushing alters this charge via the triboelectric effect. The resulting data in histogram form is shown in Fig. 10, and reveals the same trends as the mechanical data - the value and distribution of surface potential (as measured relative to the tip) is significantly modified by both treatment processes. The bleached hair data shows that the surface potential of the newly-exposed cross-linked polymer matrix is around $100 \mathrm{mV}$ more positive than the top of the fatty-acid layer (the exposed surface for the untreated hair). The surface of highlycombed hair is around $130 \mathrm{mV}$ more positive than the untreated hair, and this change will be partly triboelectric in origin and partly due to the fact that lower layers with a different chemical nature are exposed. As well as the mean value being different for each case, the spread, or standard deviation is different. It is $30 \mathrm{mV}, 25 \mathrm{mV}$ and $20 \mathrm{mV}$ for untreated, highly-combed and bleached hair, respectively, as shown in Table 2.

Table 2. Surface potential measurements - contact potential difference between tip and hair surface 


\begin{tabular}{|l|l|l|l|}
\hline & Untreated & Bleached & Highly combed \\
\hline Mean Value $(\mathrm{mV})$ & -360 & -265 & -230 \\
\hline Standard deviation $(\mathrm{mV})$ & 30 & 20 & 25 \\
\hline
\end{tabular}

This highlights the fact that this way of representing data, in histogram form rather than simply as an image, is potentially a powerful way of displaying differences between datasets that would not necessarily be clear otherwise. It also shows that simple treatments alter not only the topography, and the baseline values of elastic modulus, surface adhesion and surface potential, they also alter the distribution of each of these quantities in a way that is unique to each form of treatment. This combination of measurements could therefore be used as a "fingerprinting" technique for hair samples, revealing information about their recent history and may well be useful for forensic applications.

\section{Conclusion}

Through a combination of AFM-based imaging and nanomechanical measurements, it has been shown that simple everyday treatments such as bleaching and combing of hair can have a significant effect on the morphology and mechanical properties of hair at the nanoscale. Both forms of treatment have the effect of (i) reducing the stiffness and elastic modulus of the hair surface, as well as (ii) altering the adhesion properties of the surface, and (iii) the surface potential. We have shown that additional information may be obtained by looking not only at the mean values of each of these properties, but also their distribution. A significant result is that our measurements reveal that the variation in surface properties is consistent with what is observed at the macroscopic whole-fiber scale. 


\section{References}

[1] Bhushan, B. Biophysics of Human Hair: Structural, Nanomechanical, and Nanotribological Studies. Springer Science \& Business Media, 2010

[2] Fernandes, M. M., Lima, C. F., Loureiro, A., Gomes, A. C., and Cavaco-Paulo, A., "Keratin-based peptide: a biological evaluation and strengthening properties on relaxed hair", Int. J. Cosmet. Sci., 34, 338 (2012). doi: 10.1111/j.1468-2494.2012. 00727.x.

[3] "Guidelines to Commission Regulation (EU) No 655/2013 laying down common criteria for the justification of claims used in relation to cosmetic products | FPS Public Health," FPS Health. Available: http://www.health.belgium.be.

[4] Robbins, C. R., Chemical and Physical Behavior of Human Hair. Berlin, Heidelberg: Springer Berlin Heidelberg, 2012.

[5] Zviak, C., The Science of Hair Care. Taylor \& Francis, London, 1986.

[6] Swift, J. A., "Fine details on the surface of human hair", Int. J. Cosmet. Sci., 13, 143 (1991). doi: 10.1111/j.1467-2494.1991.tb00558.x

[7] Durkan, C. and Wang, N., "Nanometre-scale investigations iby atomic force microscopy into the effect of different treatments on the surface structure of hair", Int. J. Cosmet. Sci., 36, 598 (2014). doi: 10.1111/ics.12161

[8] Bhushan, B., Biophysics of Human Hair, Springer Berlin Heidelberg, 2010, pp. 1-19.

[9] Bhushan, B., Nanotribology and Nanomechanics, P. B. Bhushan, Ed. Springer Berlin Heidelberg, 2005, pp. 315-387.

[10] Carmen LaTorre, B. B.,"Nanotribological characterization of human hair and skin using atomic force microscopy", Ultramicroscopy, 105, $155 \quad$ (2005). doi: 10.1016/j.ultramic.2005.06.032

[11] Jachowicz, J. and McMullen, R., "Mechanical analysis of elasticity and flexibility of virgin and polymer-treated hair fibre assemblies", J. Cosmet. Sci., 53, 345 (2002).

[12] Feughelman, M., Mechanical Properties and Structure of Alpha-keratin Fibres: Wool, Human Hair and Related Fibres. UNSW Press, 1997.

[13] Smith, J. R. and Swift, J. A., "Lamellar subcomponents of the cuticular cell membrane complex of mammalian keratin fibres show friction and hardness contrast by AFM", J. Microsc., 206,182 (2002). doi: 10.1046/j.1365-2818.2002.01028.x 
[14] Smith, J. R., Tsibouklis, J., Nevell, T. G., and Breakspear, S., "AFM friction and adhesion mapping of the substructures of human hair cuticles", Appl. Surf. Sci., 285, Part B, 638 (2013). doi: 10.1016/j.apsusc.2013.08.104

[15] Wei, G., Bhushan, B., and Torgerson, P. M., "Nanomechanical chatacterization of human hair using nanoindentation and SEM", Ultramicroscopy, 105, 248, (2005). doi: 10.1016/j.ultramic.2005.06.033

[16] Krotil, H. -U., Stifter, Th. \& Marti, O., "Concurrent measurement of adhesive and elastic surface properties with a new modulation technique for scanning force microscopy", Rev. Sci. Instrum. 71, 2765 (2000). doi: 10.1063/1.1150689

[17] Smolyakov, G., Pruvost, S., Cardoso, L., Alonso, B., Belamie, E., and Duchet-Rumeau, "AFM PeakForce QNM mode: evidencing nanometre-scale mechanical properties of chitin10.1016/j.carbpol.2016.05.042

[18] Hertz, H. R., J fur die reine und angewandte Mathematik, 92, 156 (1881)

[19] Derjaguin, B. V., Muller, V. M. \& Toporov, Y. P., "Effect of contact deformations on the adhesion of particles", J. Colloid Interf. Sci. 53, 314 (1975). doi: 10.1016/0021-9797(75)90018-1

[20] Johnson, K. L., Kendall, K. \& Roberts, A. D., "Surface energy and the contact of elastic solids", Proc. R. Soc. Lond. A 324, 301 (1971). DOI: 10.1098/rspa.1971.0141

[21] Maugis, D., "Adhesion of shperes: The JKR-DMT transition using a Dugdale model", J. Colloid Interf. Sci. 150, 243 (1992). doi: 10.1016/0021-9797(92)90285-T

[22] Swift, J. A. \& Smith, J. R., "Atomic force microscopy of human hair", Scanning. 22, 310 (2000). doi: 10.1002/sca.4950220506

[23] Swift, J. A. \& Bews, B., "The chemistry of human hair cuticle-II: The isolation and amino acid analysis of the cell membranes and A-layer", J. Soc. Cosmet Chem. 25, 355 (1974). doi: 10.1.1.503.1992

[24] Gurden, S. P., Monteiro, V. F., Longo, E., and Ferreira, M. M. C., "Quantitative analysis and classification of AFM iimages of human hair", J. Microsc., 215, 13 (2004). doi: $10.1111 / \mathbf{j} .0022-2720.2004$

[25] Korte, M., Akari, S., Kühn, H., Baghdadli, N., Möhlwald, H. \& Luengo, G. S., "Distribution and localization of hydrophobic and ionic chemical groups at the surface of bleached human hair fibres", Langmuir, 30, 12124, (2014). DOI: 10.1021/1a500461y 
[26] Lodge, R. A. \& Bhushan, B., "Effect of physical wear and triboelectric interaction on surface charge as measured by Kelvin probe microscopy", J. Colloid Int. Sci. 310, 321 (2007). doi: 10.1016/j.jcis.2007.01.063

[27] Lodge, R. A. \& Bhushan, B., "Surface potential measurement of human hair using Kelvin probe microscopy", J. Vac. Sci. Technol. A 25, 893 (2007). doi: 10.1116/1.2715965

\section{Figure Captions}

Fig. 1. The structure of hair. 
Fig. 2. Evaluation of mechanical properties from the tip-sample force $(F)$ Vs distance $(D)$ relationship. The quantities that can be determined in Hybrid mode are the contact stiffness, $k$, the surface deformation, $\delta$, the adhesion force, $F_{a d h}$, the work of adhesion, $W_{a d h}$, the force, $F$, and the cantilever displacement, $D$.

Fig. 3. (a)-(c): Optical; (d)-(f): SEM and (g)-(i): AFM images showing $45 \mu \mathrm{m}$ areas of (left) untreated hair, (middle) bleached hair and (right) highly combed hair samples. The circled area in Fig. 3(f) highlights where the endocuticle has become exposed.

Fig. 4. AFM topography cross-sections of $45 \mu \mathrm{m}$ long sections of the different samples.

Fig. 5. A schematic representation of a surface profile showing descriptors used to characterize the cuticular structure, as tabulated in Table 1.

Fig. 6. AFM images of $5 \mu \mathrm{m}$ and $2 \mu \mathrm{m}$ areas of (a), (b) untreated, (c), (d) bleached and (e), (f) highly-combed hair samples.

Fig. 7. Nano mechanical properties mapped using Hybrid-mode AFM.

(a)Height (b) Stiffness (c) Adhesion lift Force for untreated hair

(d)Height (e) Stiffness (f) Adhesion lift Force for bleached hair. Circled area is for comparison of surface properties.

(g)Height (h) Stiffness (i) Adhesion lift Force for highly combed hair

(j) Cross-section along line shown in image (d); (k) molecular nature of top surface of hair, illustrating where bleach has removed some of the 18-MEA (region B) and regions that are unaffected (region A).

Fig.8. The distributions of (a) surface stiffness, (b) adhesion lift force and (c) Elastic modulus for untreated hair, bleached hair and highly combed hair revealing distinct differences as a result of the treatments each have received.

Fig.9. The distribution of surface stiffness for two extra different sets of untreated hair, bleached hair and highly combed hair showing that the same trend is observed on multiple samples.

Fig.10. (a)-(c) Topography and (d)-(f) Contact potential difference for untreated, bleached and highly combed hair, respectively; (g)-(h) Distribution of surface potential for untreated hair, bleached hair and highly combed hair for two different samples 


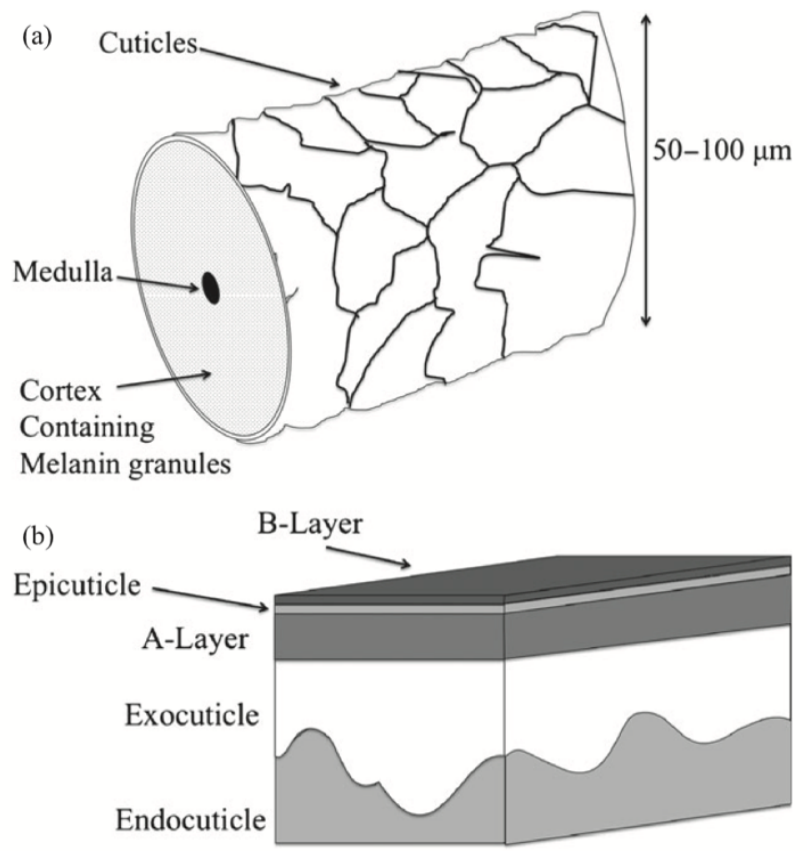

FIGURE 1. DURKAN 


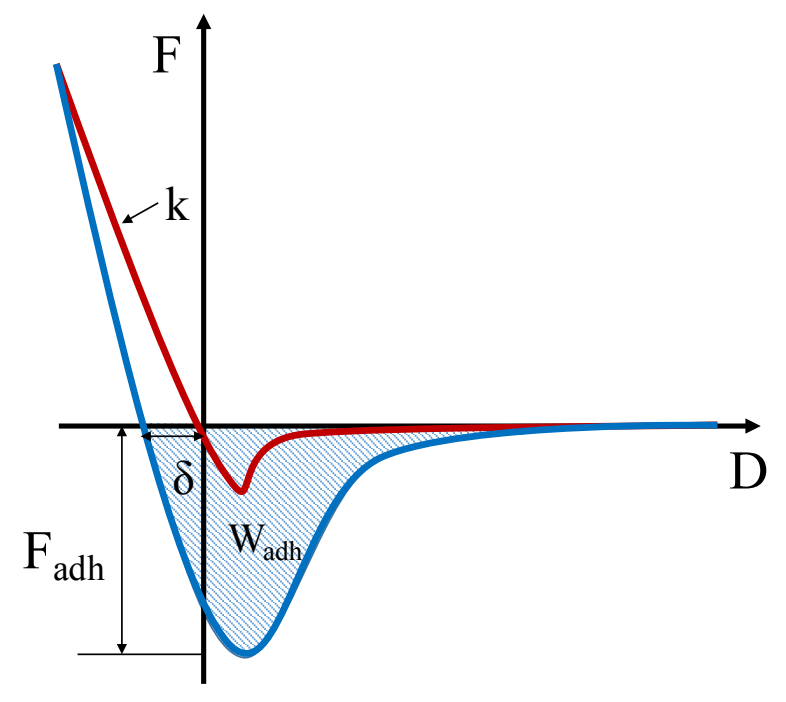

FIGURE 2. DURKAN 

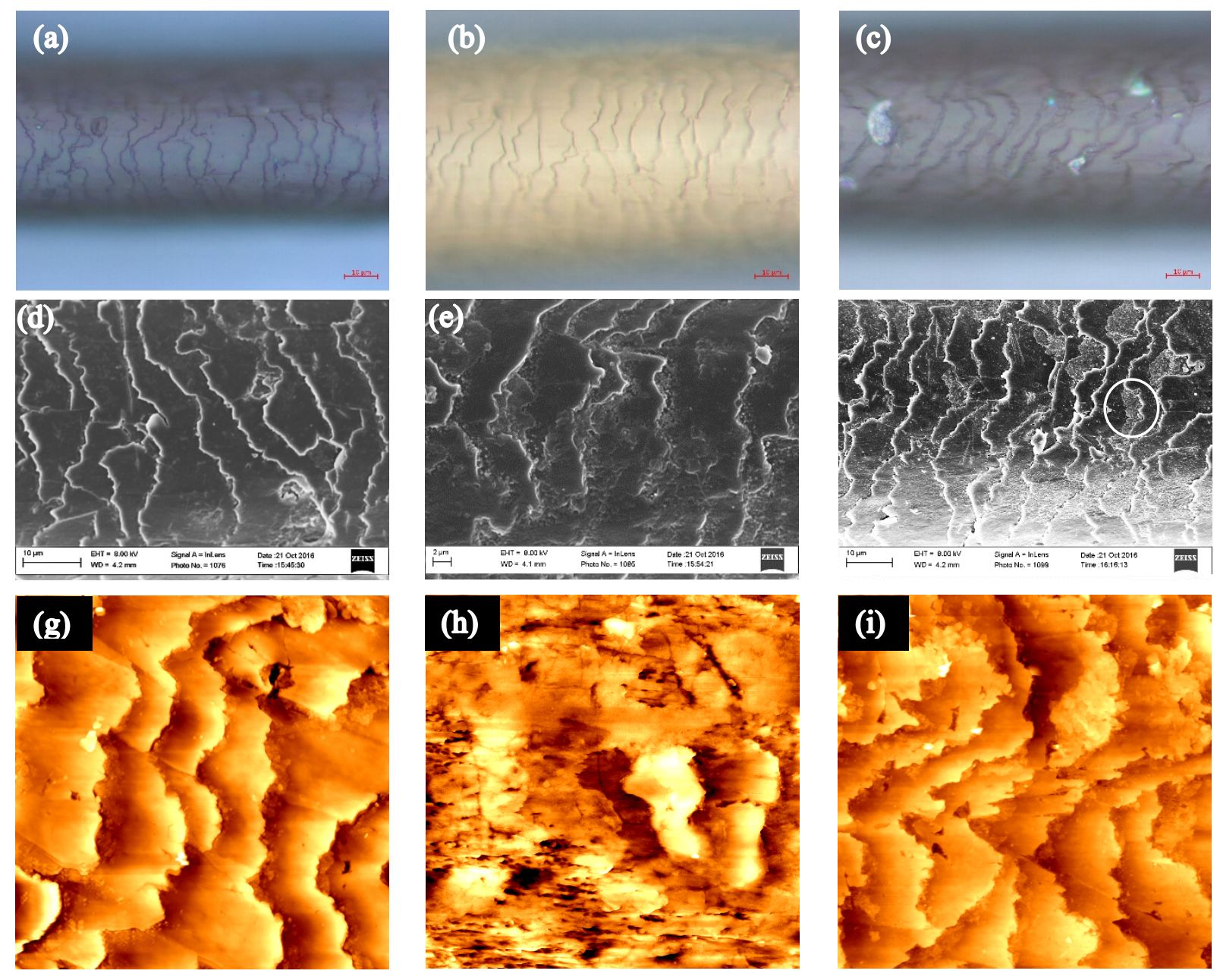

FIGURE 3. DURKAN 

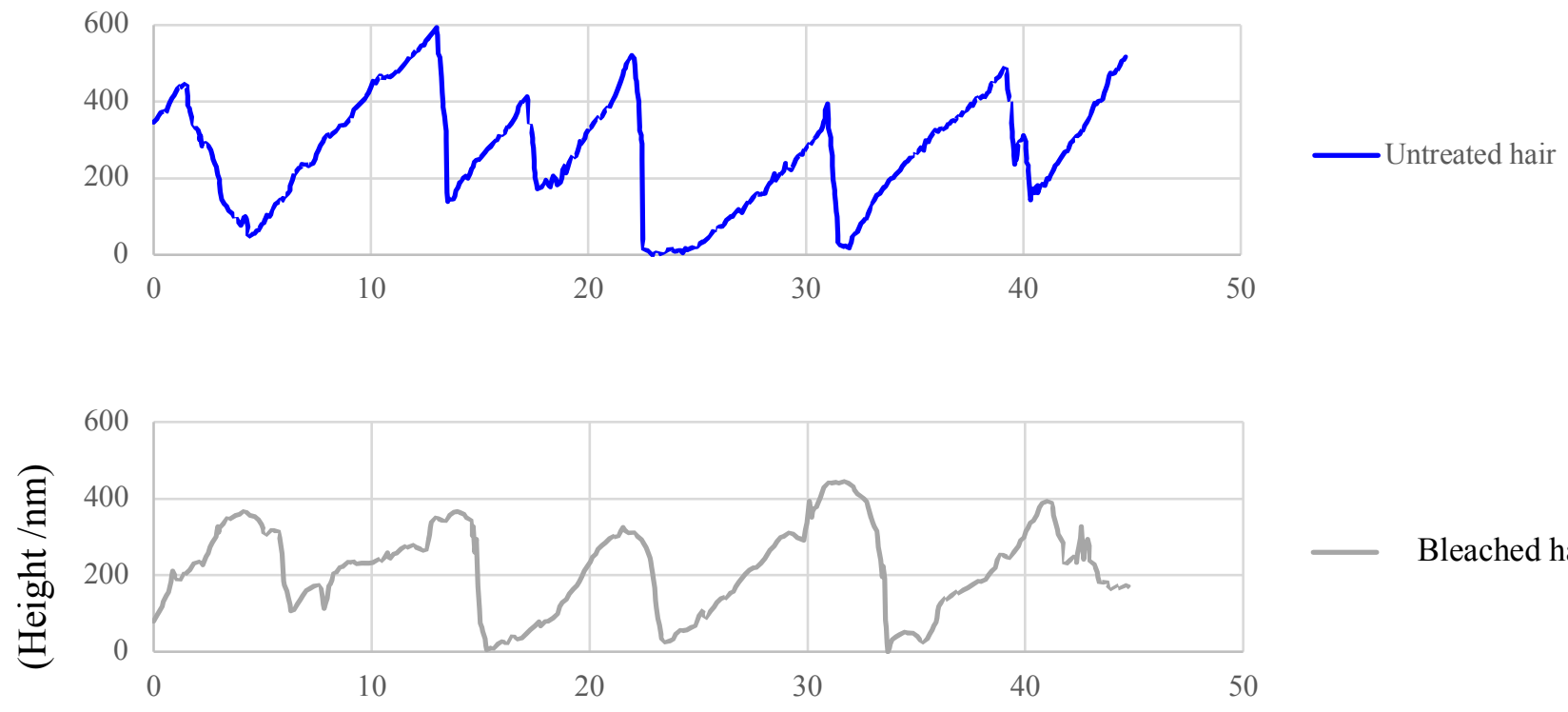

Bleached hair

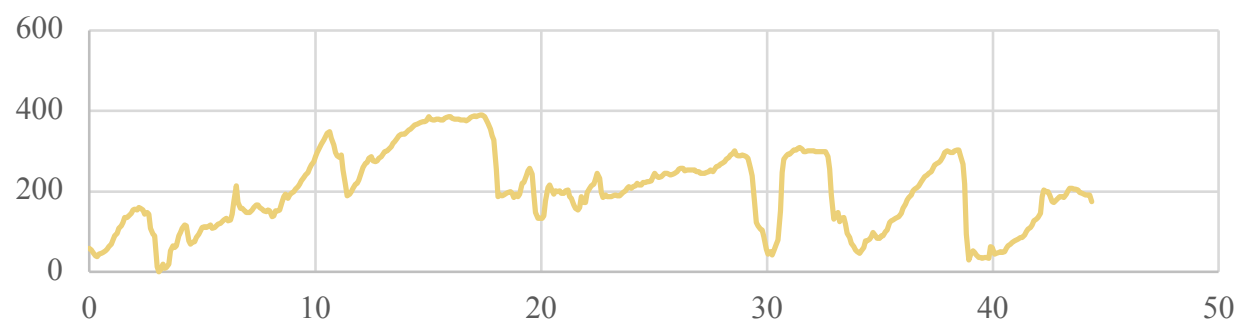

-Highly combed hair

Position / $\mu \mathrm{m}$

FIGURE 4. DURKAN 


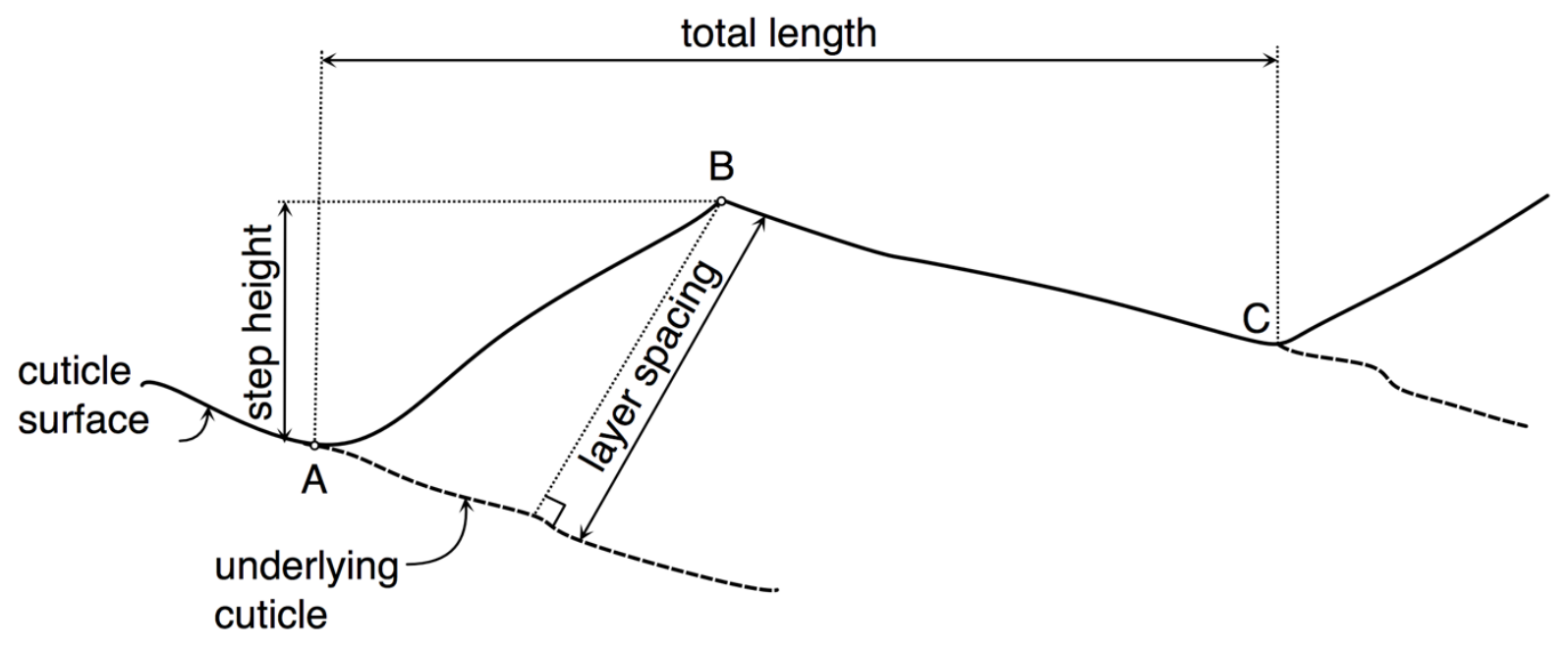

FIGURE 5. DURKAN 

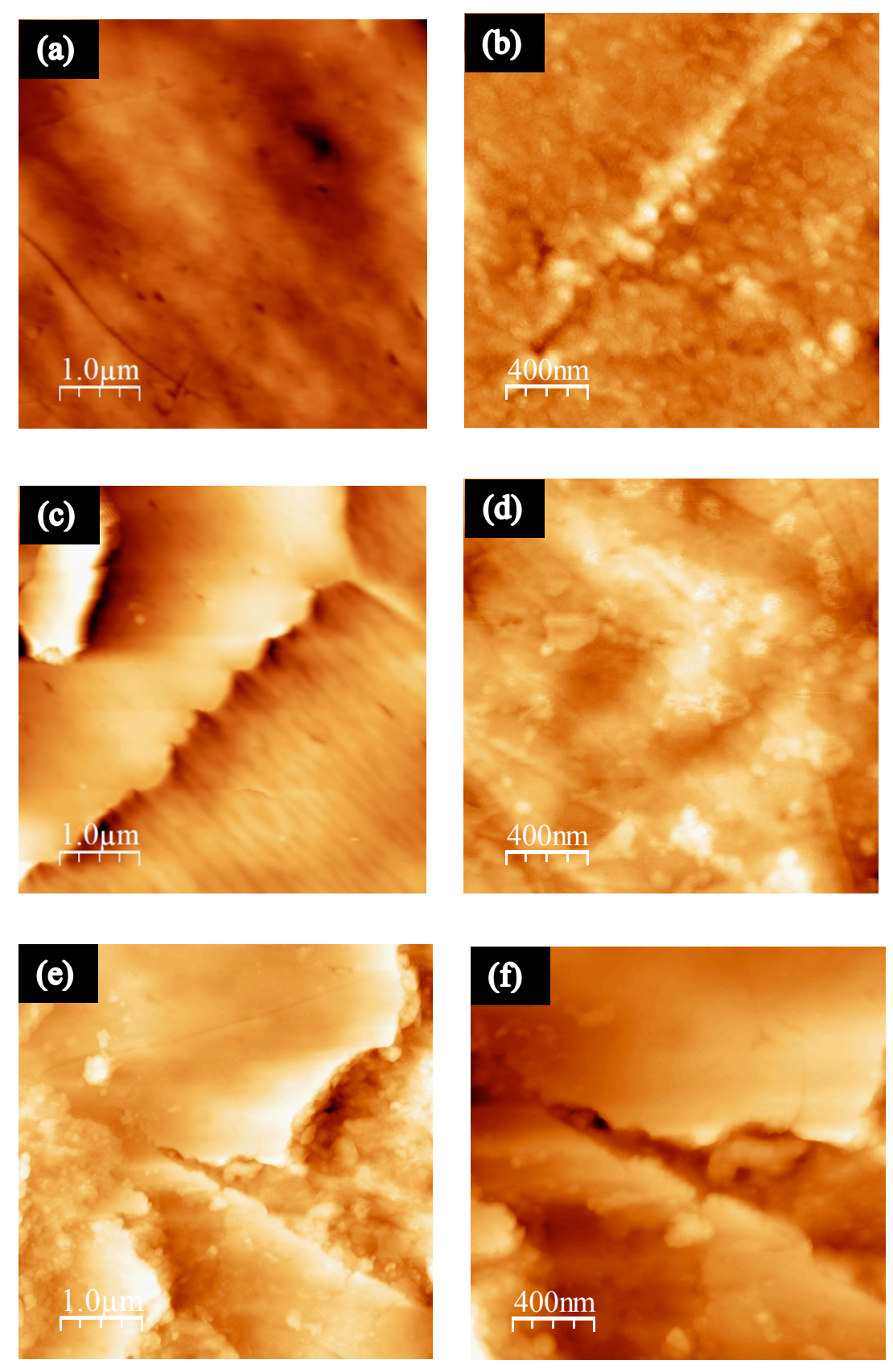

FIGURE 6. DURKAN 

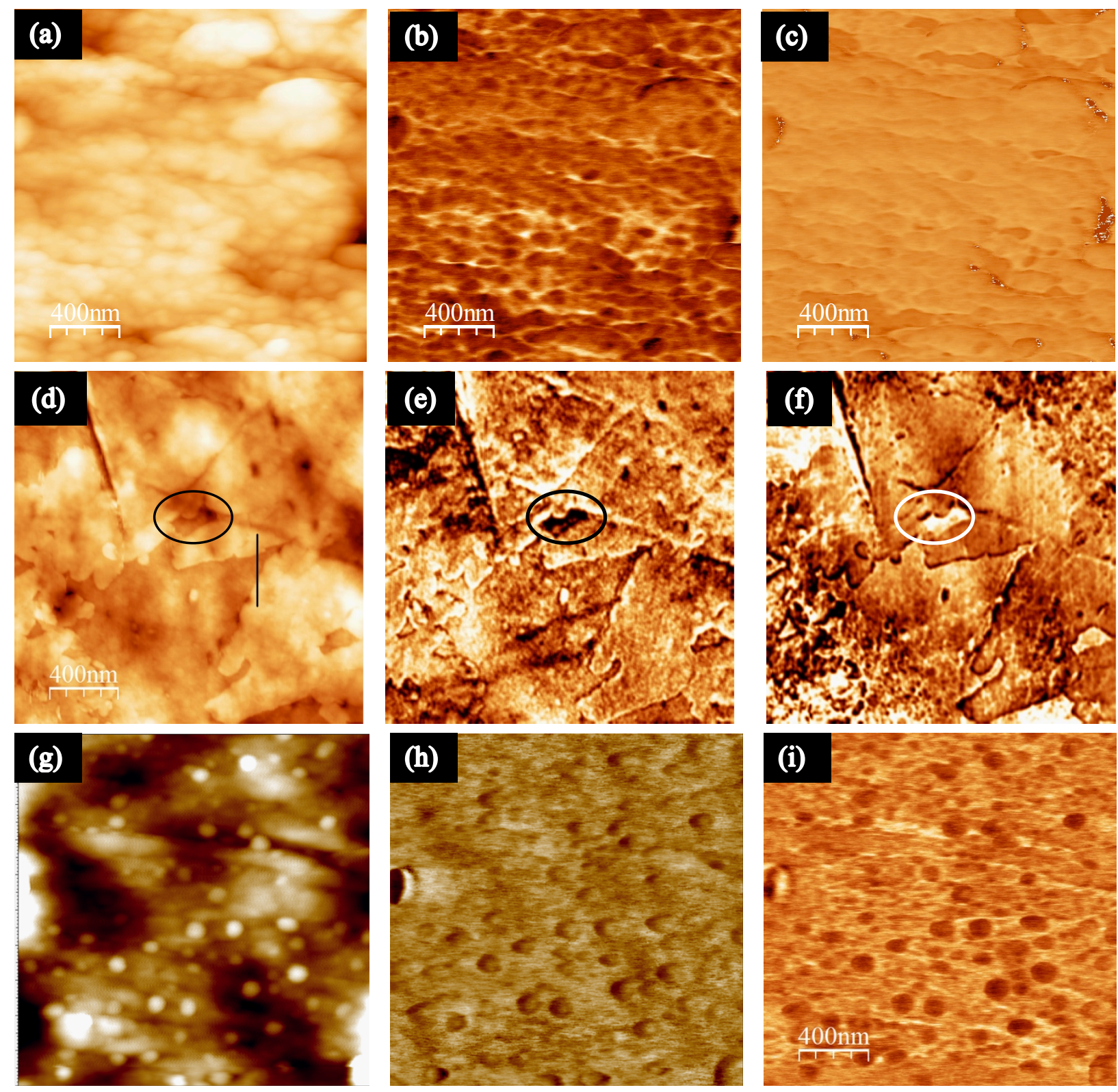

(j)

(k)
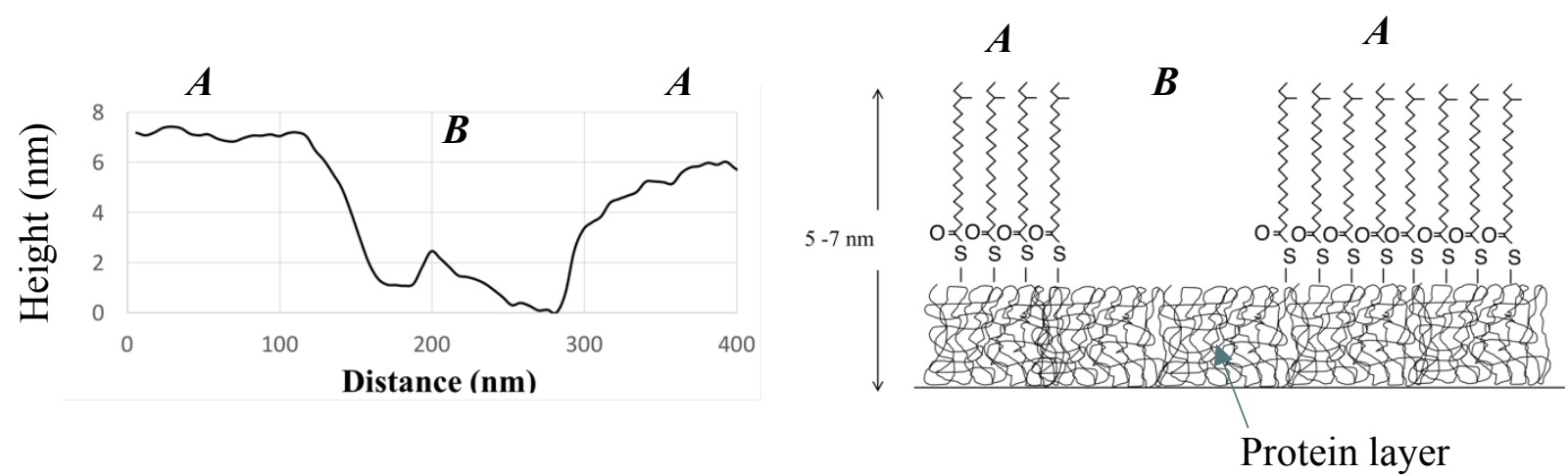

FIGURE 7. DURKAN 
(a)

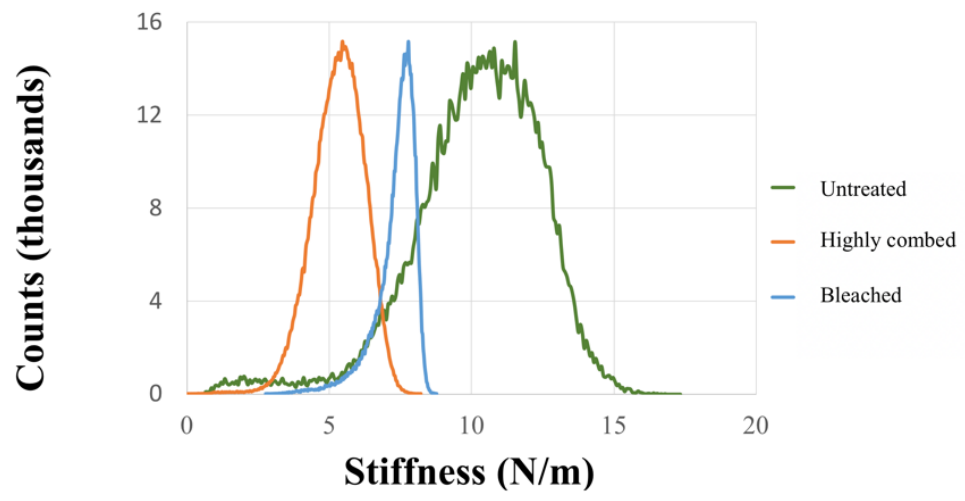

(b)

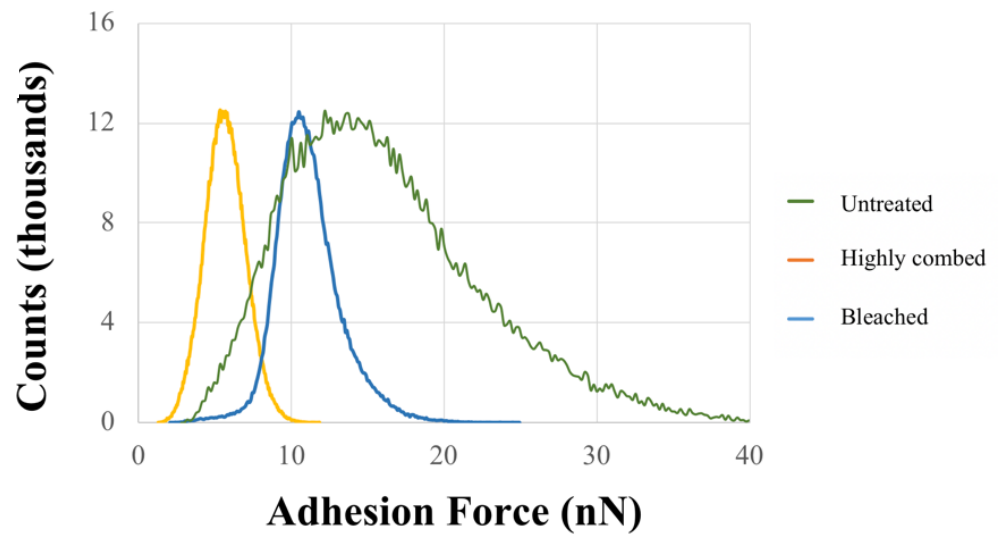

(c)

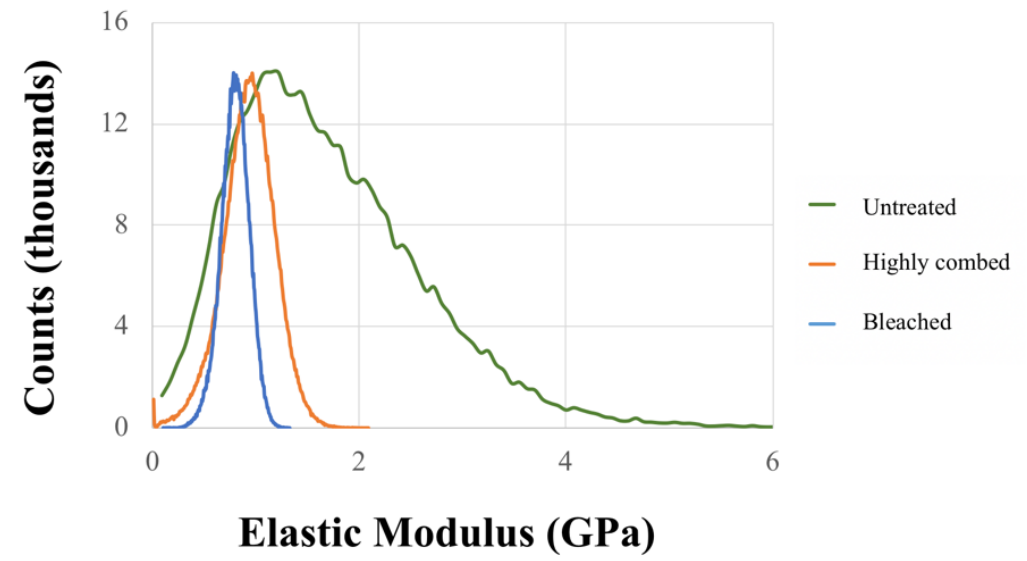

FIGURE 8. DURKAN 
(a)

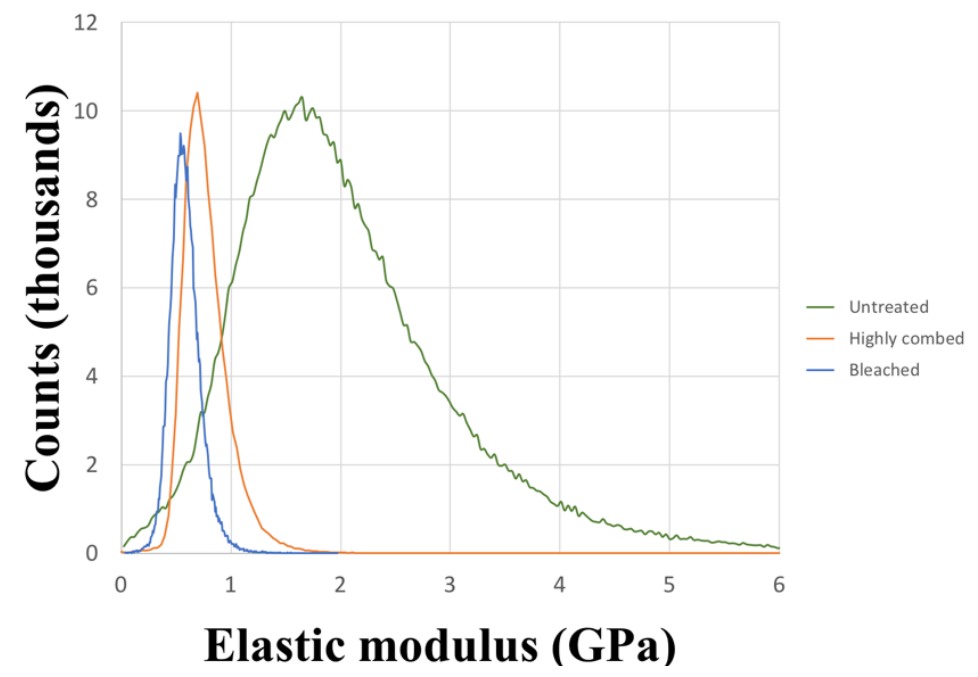

(b)

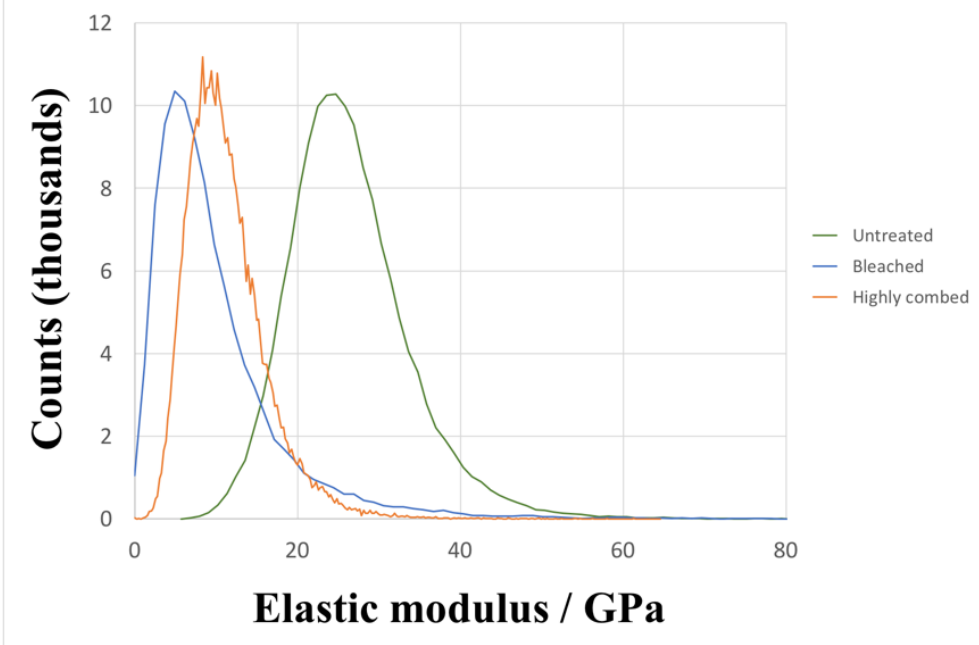

FIGURE 9. DURKAN 

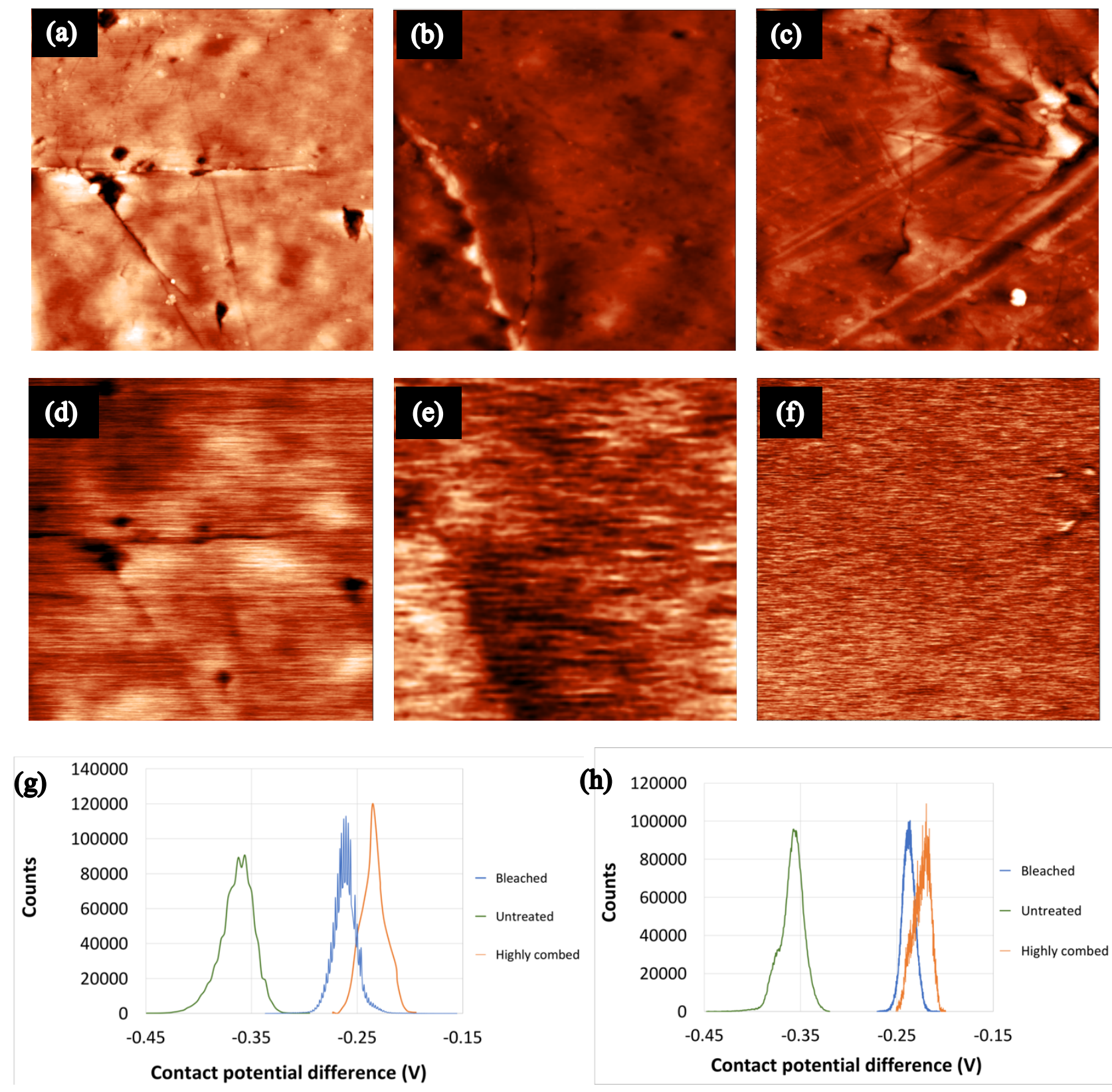

\section{FIGURE 10. DURKAN}




\section{FOR TABLE OF CONTENTS USE ONLY}

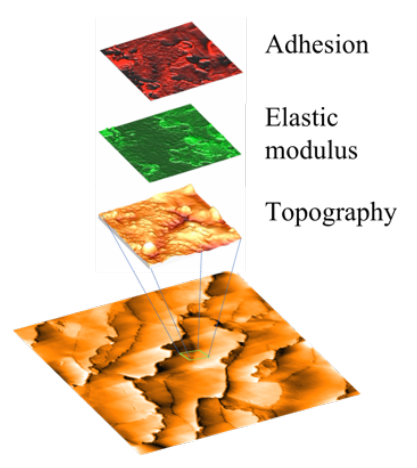

Wang, N. Barfoot, R., Butler, M. \& Durkan, C.

The effect of surface treatments on the nanomechanical properties of human hair 